\title{
Tuberculous meningitis: neuroimaging features, clinical staging and outcome
}

\begin{abstract}
Forty-two patients diagnosed with tuberculous meningitis in Kuala Lumpur Hospital based on clinical criteria, cerebrospinal fluid analysis and response to antituberculous treatment over a seven year period were included in this study. Relevant information was obtained from pa-tients' medical case notes and neuroimaging findings were evaluated. The clinical presentation of pa-tients was staged according to Medical Research Council for tuberculous meningitis. Fisher's Exact Test was used to determine the correlation between the neuroimaging features, clinical staging and outcome of patients. 52.4\% of patients had stage 2 disease, $28.6 \%$ stage 3 and the remainder stage 1 disease. $95.2 \%$ of patients had various neuroimaging abnormalities and only $4.8 \%$ had normalneuroimaging findings. The commonest neuroimaging findings were hydrocephalus and meningealenhancement. $47.6 \%$ of patients survived without any complication. 23.8\% developed morbidity ei-ther with minor or major neurological deficit and $28.6 \%$ had died at the end of the study period.Among patients with negative neuroimaging findings, one died and another one survived withoutany complication. Among patients with abnormal neuroimaging findings, 25\% developed morbidity, $27.5 \%$ died and $47.5 \%$ survived without complication. The only neuroimaging feature significantlycorrelated with clinical outcome was the presence of hydrocephalus. Therefore, hydrocephalus isimportant in the prognosis of the disease and should be considered an indicator of poor clinical out-come. There was no significant correlation between clinical staging and clinical outcome, nor wasthere a significant correlation between clinical staging and individual neuroimaging features
\end{abstract}

Keyword: Tuberculous meningitis; Neuroimaging; Clinical staging; Outcome 